\title{
BMJ Open Healthcare provider counselling for weight management behaviours among adults with overweight or obesity: a cross-sectional analysis of National Health and Nutrition Examination Survey, 2011-2018
}

\author{
Mary L. Greaney (D) , ${ }^{1}$ Steven A. Cohen, ${ }^{1}$ Furong Xu, ${ }^{2}$ Christie L Ward-Ritacco, ${ }^{2}$ \\ Deborah Riebe ${ }^{2}$
}

To cite: Greaney ML, Cohen SA, $\mathrm{Xu}$ F, et al. Healthcare provider counselling for weight management behaviours among adults with overweight or obesity: a cross-sectional analysis of National Health and Nutrition Examination Survey, 2011-2018. BMJ Open 2020;10:e039295. doi:10.1136/ bmjopen-2020-039295

- Prepublication history and additional material for this paper is available online. To view these files, please visit the journal online (http://dx.doi.org/10. 1136/bmjopen-2020-039295)

Received 15 April 2020 Revised 19 September 2020 Accepted 25 0ctober 2020
Check for updates

(c) Author(s) (or their employer(s)) 2020. Re-use permitted under CC BY-NC. No commercial re-use. See rights and permissions. Published by BMJ.

${ }^{1}$ Department of Health Studies, University of Rhode Island, Kingston, Rhode Island, USA ${ }^{2}$ Kinesiology Department, University of Rhode Island, Kingston, Rhode Island, USA

Correspondence to Dr Mary L. Greaney; mgreaney@uri.edu

\section{ABSTRACT}

Objectives To determine if adults with overweight or obesity received counselling from their healthcare providers (HCPs) to lose weight and/or adopt healthful behaviours associated with weight loss, and whether they took action on their HCPs' recommendations.

Design Cross-sectional analysis of 2011-2018 National Health and Nutrition Examination Survey (NHANES) data. Sample NHANES respondents aged $18+$ who were overweight/obese and had seen an HCP in the previous 12 months ( $n=13$ 158)

Methods Respondents reported if their HCPS recommended they control/lose weight, increase exercise/ physical activity (PA) and/or reduce fat/calorie intake, and if they adopted the offered recommendation(s). Weighted logistic regression models examined receipt of HCP counselling by sex, age, race/ethnicity, and weight status accounting for demographic characteristics and complex sampling. Similar analyses examined reported adoption of HCPs' recommendations.

Results The sample was $53.1 \%$ women, $45.0 \%$ were overweight and $55.0 \%$ had obesity. In total, $40.4 \%$ received counselling to contro//lose weight, $49.5 \%$ to increase exercise/PA and $38.9 \%$ to reduce fat/calorie intake. The following groups were less likely $(p<0.001)$ to receive counselling: men; younger adults (aged 18-39) versus middle-aged (aged 40-64) and older adults (aged $65+)$; White versus Black and Hispanic respondents; overweight respondents versus respondents with obesity. Approximately half of those advised to make changes reported doing so (53.6\% controlled/lost weight, $57.3 \%$ increased exercise/PA, $51.8 \%$ reduced fat/calorie intake). Differences in the adoption of recommendations were identified by sex, age group, race/ethnicity and weight status (all $p<0.05$ ); women, middle-aged and older adults, Black and Hispanic respondents and individuals with obesity were more likely to adopt one or more recommendations.

Conclusion Most respondents did not receive HCP counselling, and approximately half of those who received counselling reported taking action. HCPs may need training
Strengths and limitations of this study

- An important strength of this study is the use of data that are nationally representative of the US adult population.

- Additional strengths are that the study examined if respondents received healthcare provider (HCP) counselling for three weight-related recommendations (weight loss/control, increase exercise/physical activity and reduce fat/calorie intake), and also examined whether respondents acted on their HCPs' recommendations.

- Limitations of the study include the use of selfreported data to assess receipt of counselling from HCPs, and reported action on offered recommendations.

to provide counselling and to offer recommendations tailored to the social contexts of populations less likely to adopt weight control related recommendations.

\section{BACKGROUND}

Obesity is epidemic in the USA with $37.9 \%$ of men and $41.1 \%$ women having obesity. ${ }^{1}$ When estimates of overweight are included, this percentage increases to approximately $72 \% .^{1}$ The high levels of overweight and obesity are a public health crisis due to their association with increased risk of chronic disease, including cancer, diabetes, heart disease $^{2}$ and increased mortality. ${ }^{3}$

Physician counselling for weight loss is associated with increased intention to lose weight and weight loss, ${ }^{45}$ yet research indicates that more than half of patients in the USA who are overweight or have obesity have not received this counselling from their physicians. ${ }^{56}$ Analysis of data from the 2005 
National Ambulatory Medical Care Survey determined that $28.9 \%$ of adults with obesity received an obesity diagnosis, and just $17.6 \%$ received weight loss counselling. ${ }^{7}$ Among individuals who are overweight/obese, prior research has found that women are more likely than men to receive physician counselling for weight loss, ${ }^{6}$ as are individuals with higher incomes and greater educational attainment compared with individuals with lower incomes and less education. ${ }^{89}$

As energy balance and body weight are directly related, patient counselling for weight loss should include discussions related to caloric intake and expenditure. Unfortunately, the available research indicates that dietary counselling by healthcare providers (HCPs) (eg, physician, nurse practitioner, physician assistant) during medical visits rarely occurs. ${ }^{10}$ Nationally representative data from the 2011 National Health Interview Survey (NHIS) found that $32.6 \%$ of respondents received general dietary counselling from their HCPs, with Hispanic and Black respondents being more likely than White respondents, and women more likely than men, to receive dietary counselling. ${ }^{10}$ Therefore, there is a need to determine if HCPs are offering other diet-related recommendations, including limiting caloric intake, that can contribute to small deficits in energy balance and promote weight loss and/or weight maintenance in those who are overweight or have obesity.

In addition to dietary counselling, it is important that individuals with overweight or obesity receive counselling for physical activity (PA) due to its association with weight gain prevention and maintenance of weight loss. ${ }^{11} 12$ Although findings are inconsistent, ${ }^{13}$ research suggests that physician counselling to increase PA is associated with increased activity levels. ${ }^{14}{ }^{15}$ Nonetheless, HCP counselling for PA remains low. Data from 2010 indicate that only one-third of patients were counselled by their HCPs to increase PA, ${ }^{16}{ }^{17}$ and that there are differences in counselling by weight status. One study found that a greater percentage of people with normal weight $(34.4 \%)$ were advised to increase their activity compared with individuals who wereoverweight $(32.9 \%)$ or had obesity $(31.1 \%),{ }^{16}$ while another study determined that people with obesity were most likely to receive this advice. ${ }^{17}$ Moreover, women are more likely than men to receive HCP counselling for PA. ${ }^{17}$ This difference may be due to the fact that women are more likely than men to seek medical attention ${ }^{18}$ and may be more comfortable asking for advice from their HCPs than men. Research also has found that Hispanic and non-Hispanic Black adults ${ }^{17}$ and patients with higher levels of education attainment ${ }^{1617}$ are more likely to receive PA counselling.

HCP counselling for behaviour change can promote changes in behaviour. For example, receiving weight loss advice from HCPs is associated with eating less fat and calories ${ }^{1920}$ and exercising to lose weight. ${ }^{19}$ Patients with type 2 diabetes who receive weight management counselling from HCPs are more likely to report ever trying to lose weight $(89.7 \%$ vs $27.2 \%$ ) and to report modifying their diet $(85.1 \%$ vs $48.0 \%)$ compared with those who did not receive this counselling. ${ }^{21}$

There is a need to update and expand the existing research exploring weight-related counselling by HCPs and to determine if patients who are overweight or have obesity receive counselling for lifestyle behaviours associated with weight reduction and/or weight maintenance. There also is a need to determine if patients who receive this counselling take action on these recommendations. Thus, the objective of the present study was to use a nationally representative data set to: (1) explore differences in the receipt of HCP counselling to control/lose weight and/or adopt weight-related lifestyle behaviour changes by sex, age, race/ethnicity and weight status and (2) determine if there were differences in the adoption of HCPs' recommendations by sex, age, race/ethnicity and weight status.

\section{METHOD}

This study was a cross-sectional analysis of four cycles of the National Health and Nutrition Examination Survey (NHANES) data (2011-2018). NHANES uses a multistage, probability sampling to identify a sample that represent the US civilian, non-institutionalised population. See online supplemental figure 1) for the study's conceptual model. The analytical sample for the current study was limited to respondents aged 18 and older with a body mass index (BMI) of $25 \mathrm{~kg} / \mathrm{m}^{2}$ or higher who reported seeing an HCP (excluding hospital stays, emergency room and home visits and telephone calls) at least once in the 12 months prior to data collection. Of the 39156 NHANES respondents, 13158 were eligible based on our inclusion criteria and have been included in the analysis for the present study.

\section{Patient and public involvement}

Patients and the public were not involved in the design, conduct, reporting or dissemination plans of this study as the study is a secondary analysis of publicly available data.

\section{Measures}

\section{Receipt of HCP counselling}

Three items assessed receipt of HCP counselling for weight loss and lifestyle behaviours. As part of the medical conditions survey, respondents reported (yes, no) whether a doctor or health professional in the past 12 months had recommended that they: (1) control/lose weight, (2) increase exercise/PA and (3) reduce fat/calorie intake to lower their risk for certain diseases. ${ }^{22}$

\section{Action on HCP counsellingns}

Respondents who reported receiving HCP counselling for weight loss/control or lifestyle behaviours reported (yes, no) if they were: (1) trying to control/lose weight, (2) increase exercise/PA and (3) control fat/calorie intake to reduce their risk for certain diseases. ${ }^{22}$ 


\section{Covariates}

Covariates were selected based on the available literature and the focus of the paper and included weight status, age, sex, race/ethnicity, PA and income to poverty ratio (PIR). BMI was calculated using height and weight measured by trained NHANES staff (20). BMI was then used to determine weight status (overweight $\left(25-29.9 \mathrm{~kg} / \mathrm{m}^{2}\right)$ or obese $\left.\left(\geq 30 \mathrm{~kg} / \mathrm{m}^{2}\right)\right){ }^{23}$ Respondents also reported their age, sex, race/ethnicity (White, Black, Hispanic (MexicanAmerican and other Hispanic), other), education (high school diploma or less, some college, college degree or above) and smoking status (never, former, current). For analysis, age was categorised and used to create the following groups: younger adults (aged 18-39), middleaged adults (aged 40-64) and older adults (65 years of age or older). Family income and family size were used to calculate the PIR, the ratio of family income to the federal poverty level, ${ }^{22}$ which was then dichotomised to at/above or below the poverty level.

Self-reported PA was measured using the Global Physical Activity Questionnaire, a 16-item instrument that assesses frequency and duration of work-related, travelrelated and leisure time $\mathrm{PA} .{ }^{24} \mathrm{~A}$ score was calculated for each PA category and scores were summed to calculate daily minutes of moderate to vigorous PA. ${ }^{24}$ Participants were then classified as to whether they met the current aerobic PA recommendation (yes vs no) using the US Department of Health and Human Services' recommendation of at least $150 \mathrm{~min} /$ week of moderate or $75 \mathrm{~min} /$ week vigorous intensity $\mathrm{PA}$ or an equivalent combination of both. $^{25}$

\section{Statistical analyses}

Based on the NHANES Analytic Guideline for sample weights selection and construction when using multiple NHANES survey cycles, ${ }^{26}$ the NHANES mobile examination centre exam 8-year weights were used for all analyses in an effort to address complex survey design. Descriptive results were assessed with means \pm SE being calculated for continuous variables and frequencies and weight proportions (n (weighted \%)) for categorical variables. $\mathrm{P}$ values were obtained by performing linear regression and logistic regression analyses for categorical and continues variables, respectively. Weighted multivariate logistic regression models were used to calculate adjusted odds ratios (ORs) and 95\% CIs, and to determine if there were differences in being advised by HCPs to: (1) control/lose weight, (2) increase exercise/PA and (3) reduce fat/calorie intake, by sex, race/ethnicity, age group and weight status. The models were adjusted for age, sex, education, BMI, PIR, smoking status and PA, with covariates being added to the model simultaneously. Similarly, weighted multivariate logistic regression models, also adjusted for age, sex, education level, BMI, PIR, smoking status and PA, were constructed to examine differences in reported action on HCP counselling by sex, age, race/ethnicity and weight status among respondents who reported being offered the corresponding behaviour counselling. Respondents with missing data on key variables were excluded from the analysis. All analyses were conducted using SAS V.9.4 (SAS Institute Inc., Cary, NC) with $\mathrm{p}<0.05$ set a priori for statistical significance.

\section{RESULTS}

The analytic sample included 13158 respondents. The average age for respondents was 49.8 years, and $53.1 \%$ were women, $36.0 \%$ had a high school education or less, $45.0 \%$ were overweight and $55.0 \%$ had obesity. Furthermore, $58.1 \%$ of respondents were classified as meeting the current aerobic PA recommendation. There were statistically significant differences between racial/ethnic groups regarding percentage of men, educational attainment and household income (see table 1 ).

\section{Receipt of HCP counselling}

The percentage of respondents who reported receiving HCP counselling to control/lose weight, increase exercise/PA and reduce fat/calorie intake was $40.4 \%, 49.5 \%$ and $38.9 \%$, respectively (see figure 1 and online supplemental table 1 ). In total, $19.6 \%$ of overweight individuals and $57.5 \%$ of individuals with obesity reported receiving HCP counselling to control/lose weight in the previous 12 months. As shown in table 2, women were more likely than men to report being counselled to control/lose weight $(\mathrm{OR}=1.21,95 \%$ CI: $1.10,1.32)$, increase exercise/ PA (OR=1.34, 95\% CI: 1.22, 1.46) and reduce fact/calorie intake $(\mathrm{OR}=1.13,95 \% \mathrm{CI}: 1.00,1.27)$.

Differences in HCP counselling by age were identified. Middle-aged adults (aged 40-64) were more likely than younger adults (aged 18-39) to receive HCP counselling to control/lose weight ( $\mathrm{OR}=1.72,95 \% \mathrm{CI}: 1.53,1.93)$, increase exercise $/ \mathrm{PA} \quad(\mathrm{OR}=1.71,95 \%$ CI: $1.49,1.97)$ and reduce fat/calorie intake $(\mathrm{OR}=2.00,95 \% \mathrm{CI}: 1.76$, 2.28). A similar pattern was observed between younger and older adults (aged 65+), with older adults having an increased likelihood of being advised to control/ lose weight, increase exercise/PA and reduce fat/calorie intake (see table 2).

Additionally, differences in counselling by race/ ethnicity and weight status were identified. Black respondents were more likely than White respondents to report being advised to control/lose weight $(\mathrm{OR}=1.26,95 \% \mathrm{CI}$ : $1.11,1.44)$, increase exercise $/ \mathrm{PA}(\mathrm{OR}=1.25,95 \% \mathrm{CI}: 1.10$, $1.43)$ and reduce fat/calorie intake $(\mathrm{OR}=1.49,95 \% \mathrm{CI}$ : $1.29,1.71)$. Hispanic respondents were more likely than White respondents to be receive counselling as were individuals with obesity (see table 2 ).

\section{Reported action on HCPs' recommendations}

Approximately half of respondents who received counselling from their HCPs to control/lose weight and/or change their behaviours reported that they acted on these recommendations (see figure 2 and online supplemental table 2). More specifically, among those who received counselling, $53.6 \%$ reported that they had controlled/ 
Table 1 Characteristics of respondents aged 18+ by weight status, and differences by weight status NHANES 2011-2018 $(n=13158)$

\begin{tabular}{|c|c|c|c|c|}
\hline Variables & $\begin{array}{l}\text { Total } \\
(n=13 \text { 158) } \\
n \text { (weighted \%) }\end{array}$ & $\begin{array}{l}\text { Overweight } \\
\text { ( } n=5876,45.0 \%) \\
n \text { (weighted \%) }\end{array}$ & $\begin{array}{l}\text { Obese } \\
\text { ( } n=7282,55.0 \%) \\
n \text { (weighted \%) }\end{array}$ & $P$ value \\
\hline \multicolumn{5}{|l|}{ Sex } \\
\hline Male & 6015 (46.9) & $3055(51.4)$ & 2960 (43.2) & $<0.001^{*}$ \\
\hline Female & $7143(53.1)$ & $2821(48.6)$ & $4322(56.8)$ & $<0.001^{*}$ \\
\hline Age (years) & $49.8 \pm 0.3$ & $50.1 \pm 0.4$ & $49.5 \pm 0.3$ & 0.119 \\
\hline 18-39 & $3821(30.7)$ & $1718(31.3)$ & $2103(30.2)$ & 0.351 \\
\hline $40-64$ & $5849(47.1)$ & $2440(44.6)$ & 3409 (49.1) & $0.001^{*}$ \\
\hline $65+$ & 3488 (22.2) & $1718(24.2)$ & $1770(20.6)$ & $0.001^{*}$ \\
\hline \multicolumn{5}{|l|}{ Education level } \\
\hline High school diploma or less & $5593(36.0)$ & $2422(34.0)$ & $3171(37.7)$ & $<0.001^{*}$ \\
\hline Some college or more & 7149 (64.0) & $3243(66.0)$ & 3906 (62.3) & $<0.001^{*}$ \\
\hline \multicolumn{5}{|l|}{ Race/ethnicity } \\
\hline White & $5002(66.3)$ & $2279(67.8)$ & $2723(65.1)$ & $0.02^{\star}$ \\
\hline Black & 3327 (12.3) & $1208(9.6)$ & 2119 (14.5) & $<0.001^{*}$ \\
\hline Hispanic & $3293(14.5)$ & $1437(14.0)$ & $1856(14.8)$ & 0.135 \\
\hline Other & $1536(6.9)$ & $952(8.6)$ & $584(5.5)$ & $<0.001^{*}$ \\
\hline$P I R \geq 1.0$ & 9297 (86.2) & $4216(87.5)$ & $5081(85.2)$ & $0.003^{*}$ \\
\hline \multicolumn{5}{|l|}{ Smoking status } \\
\hline Never smoked & 7525 (56.5) & $3376(57.3)$ & $4149(55.8)$ & 0.269 \\
\hline Formerly smoked & $3361(27.4)$ & $1465(26.4)$ & $1896(28.1)$ & 0.135 \\
\hline Currently smoke & $2161(16.1)$ & $977(16.3)$ & $1184(16.0)$ & 0.786 \\
\hline BMI $\left(\mathrm{kg} / \mathrm{m}^{2}\right)$, mean (SE) & $32.2 \pm 0.1$ & $27.4 \pm 0.0$ & $36.2 \pm 0.1$ & $<0.001^{*}$ \\
\hline Met PA recommendation, $\ddagger \mathrm{n}$ & $6588(58.1)$ & 3077 (62.5) & $3511(54.4)$ & $<0.001^{*}$ \\
\hline
\end{tabular}

Overweight=BMI of $25.0-29.9 \mathrm{~kg} / \mathrm{m}^{2}$; Obesity=BMI of $30+\mathrm{kg} / \mathrm{m}^{2}$. Data are presented as weighted mean $\pm S E$ for continuous variables and frequencies and weight proportions for categorical variables.

${ }^{*} \mathrm{p}<0.05$.

¥PA recommendation=at least $150 \mathrm{~min} /$ week of moderate or $75 \mathrm{~min} /$ week vigorous intensity PA, or an equivalent combination of both. BMI, body mass index; NHANES, National Health and Nutrition Examination Survey; PA, physical activity; PIR, ratio of family income to poverty.

lost weight, $57.3 \%$ said that they increased exercise/PA and $51.8 \%$ indicated they had reduced fat/calorie intake.

Women were more likely than men to report adopting the recommendation to increase exercise $/ \mathrm{PA}(\mathrm{OR}=1.43$, 95\% CI: $1.21,1.67)$. There was, however, no statistically significant difference between men and women in reported efforts to control/lose weight or reduce fat/ calorie intake. Middle-aged ( $\mathrm{OR}=0.83,95 \%$ CI: 0.71 , 0.98 ) and older adults (OR=0.66, 95\% CI: 0.54, 0.81) were less likely to report increasing exercise/PA than younger adults, but were more likely to report reducing fat/calorie intake than younger adults (middle-aged: ( $\mathrm{OR}=1.49,95 \%$ CI: $1.27,1.74)$; older adults $(\mathrm{OR}=1.37$, 95\% CI: $1.10,1.71)$ ).

As shown in table 3 , there were differences in the adoption of HCPs' recommendations by race/ethnicity and weight status. Black respondents were more likely than White respondents to report adopting HCPs' recommendations (control/lose weight $(\mathrm{OR}=1.23,95 \%$ CI: 1.08 ,
1.40), increase exercise /PA (OR=1.34, 95\% CI: 1.14, 1.57) and reduce fat/calorie intake $(\mathrm{OR}=1.44,95 \%$ CI: 1.25 , 1.66)). Hispanic respondents were also more likely than White respondents to report adopting the recommendation to control/lose weight and to reduce fat/calorie intake. There was no difference between Hispanic and White respondents in terms of reported efforts to increase exercise/PA. Furthermore, respondents classified as having obesity were more likely to adopt HCPs' recommendations than respondents who were overweight.

\section{DISCUSSION}

In this nationally representative sample of adults aged $18+$ who were overweight or had obesity, $19.6 \%$ of overweight individuals and $57.5 \%$ of individuals with obesity reported receiving HCP counselling to control or lose weight in the previous 12 months. These findings align somewhat with prior studies. ${ }^{8}$ For example, $44 \%$ of respondents 


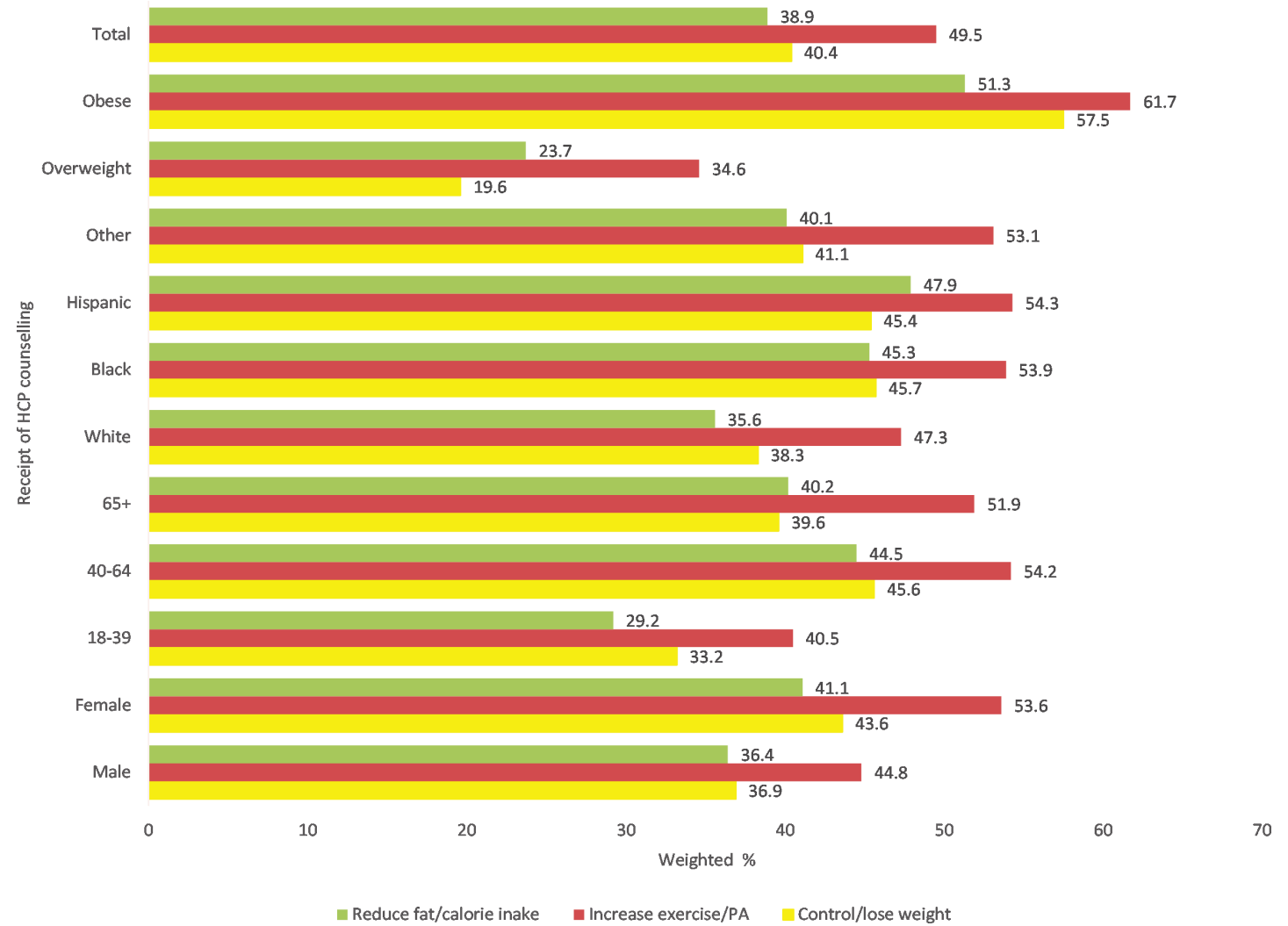

Figure 1 Receipt of healthcare provider (HCP) counselling by sex, age, race/ethnicity and weight status ( $n=13$ 158). Note: PA = physical activity.

with obesity were advised by HCPs to lose weight in 1994; this decreased to $40 \%$ in $2000 .{ }^{9}$ More recently, Lorts and Ohri-Vachaspati found that $42 \%$ of those with a BMI of $30.0-39.9 \mathrm{~kg} / \mathrm{m}^{2}$ and $63 \%$ of those with a BMI $\geqq 40 \mathrm{~kg} /$ $\mathrm{m}^{2}$ were advised by their HCPs to lose weight. ${ }^{8}$ While the percentage of individuals with obesity counselled to lose/ control weight found in the current study is encouraging, it is concerning that only $19.6 \%$ of individuals who were overweight received this advice. The health risks associated with overweight are well documented, and there is evidence to support that being overweight increases one's risk for developing obesity. ${ }^{2}$ It is not clear why individuals who were overweight were not counselled to lose/control weight: however, it is possible that the HCPs do not view being overweight as problematic as being obese. ${ }^{27}$

In the current study, $61.7 \%$ of respondents with obesity were advised to increase exercise/PA. An analysis of data from the 2010 NHIS data determined that $30.5 \%$ of overweight and $46.9 \%$ of respondents with obesity had received similar advice. ${ }^{16}$ The increase in HCP counselling may be due to increased knowledge among HCPs about the importance of PA for prevention of a growing number of health conditions and/or the recent release of the second edition of the Physical Activity Guidelines for Americans, ${ }^{25}$ which aims to increase the ease of relaying public health recommendations for PA. Although the present study found that a greater percentage of patients received HCP counselling to increase exercise/PA than prior work, ${ }^{16}$ more patients should have received this advice, as only $58.1 \%$ were meeting the current aerobic PA recommendation based on self-reported activity levels.

Results of the current study indicate that HCPs were more likely to counsel patients to increase exercise/PA over reducing caloric/fat intake and/or controlling/ losing weight. Only $38.9 \%$ of respondents reported being advised to reduce fat/calorie intake $23.7 \%$ of respondents with overweight, $51.3 \%$ of respondents with obesity), which is similar to, ${ }^{10}$ and greater than rates found in prior studies. ${ }^{728}$ It is possible that HCPs offer recommendations to increase exercise/PA over weight control/loss, as they may be hesitant to discuss body weight with patients due the potentially sensitive nature of this topic. ${ }^{29}$ Additionally, HCPs may not provide nutrition counselling due a lack of training, low self-efficacy to provide this counselling, ${ }^{30}$ and limited time. ${ }^{31}$ Future research could explore why dietary changes are not being regularly recommended.

The current study identified gender differences in the receipt of HCP counselling, with a greater percentage of women than men being advised to control/lose weight, increase exercise/PA and reduce fat/calorie intake. This finding confirms prior research. ${ }^{8}{ }^{10}{ }^{16}$ It is possible that HCPs perceive women as being more receptive than men to weight loss-related recommendations and advice. Additionally, women visit HCPs more often ${ }^{32}$ and as a result may have developed a more trusting relationship with their HCPs, allowing for more sensitive conversations about weight-related topics. 
Table 2 Comparative ORs of receipt of healthcare provider (HCP) counselling by sex, age, race/ethnicity and weight status $(n=13$ 158)

\begin{tabular}{|c|c|c|c|c|c|c|}
\hline & \multicolumn{6}{|l|}{ HCP counselling to } \\
\hline & \multicolumn{2}{|l|}{ Control/lose weight } & \multicolumn{2}{|l|}{ Increase exercise/PA } & \multicolumn{2}{|c|}{ Reduce fat/calorie intake } \\
\hline & Adjusted OR (95\% CI) & $P$ value & Adjusted OR (95\% Cl) & $P$ value & Adjusted OR (95\% Cl) & $\mathbf{P}$ value \\
\hline Male & Ref & & Ref & & Ref & \\
\hline Female & 1.21 (1.10 to 1.32$)$ & & 1.34 (1.22 to 1.46$)$ & & 1.13 (1.00 to 1.27$)$ & \\
\hline 18-39 & Ref & & Ref & & Ref & \\
\hline $40-64$ & $1.72(1.53$ to 1.93$)$ & & 1.71 (1.49 to 1.97$)$ & & 2.00 (1.76 to 2.28$)$ & \\
\hline $65+$ & $1.48(1.27$ to 1.71$)$ & & 1.65 (1.39 to 1.96$)$ & & 1.85 (1.55 to 2.20$)$ & \\
\hline Race/ethnicity & & $<0.001^{*}$ & & $<0.001^{*}$ & & $<0.001^{\star}$ \\
\hline White & Ref & & Ref & & Ref & \\
\hline Weight status & & $<0.001^{*}$ & & $<0.001^{*}$ & & $<0.001^{\star}$ \\
\hline Overweight & Ref & & Ref & & Ref & \\
\hline Obese & 5.56 (4.87 to 6.34$)$ & & 3.00 (2.59 to 3.47$)$ & & 3.35 (2.97 to 3.79$)$ & \\
\hline
\end{tabular}

Overweight=BMI of $25.0-29.9 \mathrm{~kg} / \mathrm{m}^{2}$; Obesity=BMl of $30+\mathrm{kg} / \mathrm{m}^{2}$. Adjusted odds ratios (ORs) were obtained by performing multivariate PROC SURVEYLOGISTIC procedure, adjusted for all the variables in the table, and education level, PIR (ratio of family income to poverty), smoking status and physical activity.

${ }^{*} \mathrm{p}<0.05$.

BMI, body mass index; PA, physical activity.

Age also may be a factor of consideration affecting HCPs' decisions to recommend lifestyle changes related to weight loss. In the current study, younger adults were less likely than middle-aged or older adults to received HCP counselling. This finding corresponds with prior research, ${ }^{10} 1633-35$ and warrants concern, as the adoption of healthy lifestyle behaviours earlier in life may help reduce their risk of developing chronic conditions associated with overweight and obesity. ${ }^{36}$ Intentional weight loss by older adults with obesity is associated with improved cardiovascular health, reduced inflammation and improved health-related quality of life, while being overweight may offer protection against wasting and other related disorders among older adults, especially the oldest old. ${ }^{37} 38$ These contrasting effects of excess weight can be confusing to HCPs and patients, and may contribute to the current rates of weight loss-related counselling associated with HCP visits. Additional research is needed to understand why HCPs are less likely to advise younger adults to lose weight.

The current study also identified differences in HCP counselling by race/ethnicity. Black and Hispanic respondents were more likely than White respondents to report receiving HCP counselling. Analysis of 2010 NHIS data found that Hispanic and Black respondents were less likely to receive diet-related advice from HCPs than White respondents; however, the 2011 data determined that Hispanic and Black respondents were more likely than Whites respondents to receive this advice. ${ }^{10}$ Reasons for these increase could be heighted awareness among by HCPs that overweight and obesity rates are higher among Blacks and Hispanics than Whites, as recent data from the National Center for Health Statistics reported that Hispanics (47.0\%) and non-Hispanic Blacks (46.8\%) had the highest prevalence of obesity, followed by nonHispanic Whites (37.9\%) and non-Hispanic Asians $(12.7 \%){ }^{39}$

Additional research is needed to understand why HCPs do not offer counselling to more patients with overweight and obesity. While time constraints, limited training on counselling, and low self-efficacy may serve as barriers, ${ }^{40-45}$ recently, it has been noted that medical schools in the USA are not adequately preparing physicians to manage patients with obesity. ${ }^{46}$ Initial training and continuing education for HCPs should address these issues in a more comprehensive manner and help HCPs recognise the need for increased counselling related to weight loss and weight-related behaviours. HCPs may also need training on identifying patients who are overweight, ${ }^{47} 48$ and recognising possible bias they may have regarding treatment for obesity as being ineffective. ${ }^{49}$ It has been recommended that HCPs have an open discussion with their patients' about their personal health concerns, factors affecting their health status, and discuss the risks 


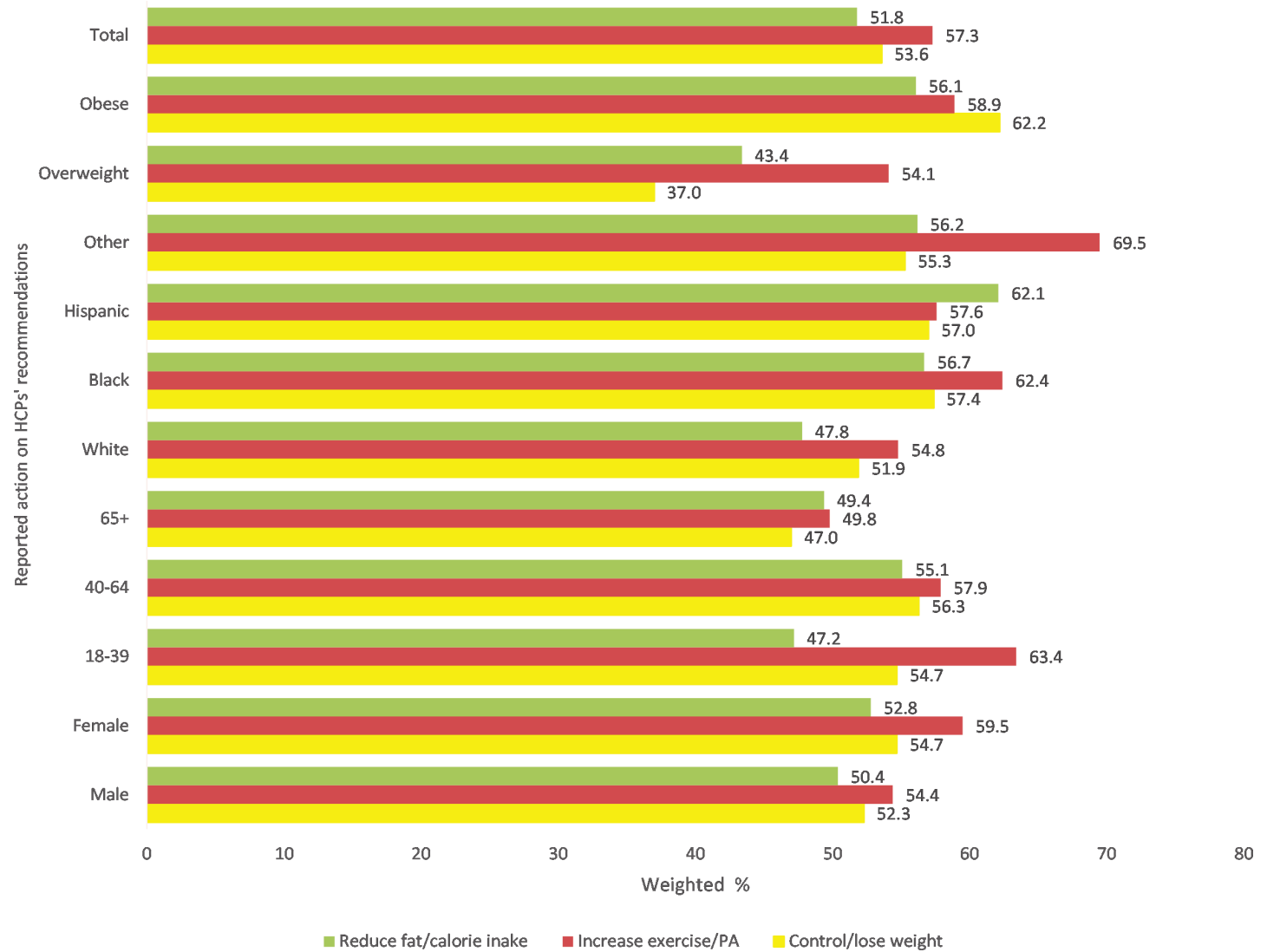

Figure 2 Reported action on healthcare providers' (HCPs') recommendations by sex, age, race/ethnicity and weight status $(\mathrm{n}=8458)$. Note: $\mathrm{PA}=$ physical activity.

associated with overweight/obesity before addressing the patient's weight status. ${ }^{50}$ The use of PA as a vital sign has been recommended. ${ }^{40}$ This clinical tool encourages HCPs to ask patients about their PA habits at each visit and allows for tracking of PA levels by incorporating results into electronic medical records. This would make the discussion about PA easier and more likely to occur.

In addition to examining the receipt of HCP counselling, the current study explored whether respondents who were counselled by HCPs acted on the offered advice. Between $51.8 \%$ and $57.3 \%$ of participants who received HCP counselling reported taking action on the offered advice (control/lose weight, increasing exercise/PA and/or reducing fat/calorie intake). These numbers are encouraging and support the idea that HCP counselling is valuable in promoting behaviour change. Women were more likely than men to reported increasing exercise/PA, but no other gender differences were identified in the adoption of the other recommendations. Differences in reported action by age also were identified, with middleaged adults and older adults being less likely to report that they had increased exercise/PA and more likely to say they had limited fat/calories than younger adults. This finding suggests that HCPs should discuss the importance of healthful behaviours across the lifespan and may need to help middle-aged and older adults incorporate PA into their life. It is possible that some PA advice offered by HCPs does not resonate with older adults (eg, join a gym) or that older adults may have lower self-efficacy to be physically active, supporting the idea that PA advice needs to be personalised.

The present study also found differences in reported adoption of recommendations by race/ethnicity and weight status. Black and Hispanic respondents were more likely than White respondents to report they had adopted advice to control/lose weight, limit fat/calories and/or increase exercise/PA. Respondents with obesity were more likely to adopt the recommendations than overweight respondents. It is possible the individuals with obesity were motivated to adopt offered recommendations due to a heightened awareness of health risks associated with obesity or that HCPs offer individuals with obesity more thorough counselling than what is offered to respondents who are overweight. These finding indicate HCPs should continue to recommend that patients with obesity control/lose weight, increase exercise/PA and reduce fat and calorie intake, as these recommendations seem to be considered and adopted.

HCPs should provide patients with appropriate referrals. A study determined that adults with obesity who were randomised to receive a referral from their primary care provider to weight loss treatment were more likely to initiate treatment than those randomised to usual care. ${ }^{51}$ The American College of Sports Medicine encourages primary care physicians to include PA as part of integrative treatment plans by assessing PA at every healthcare 


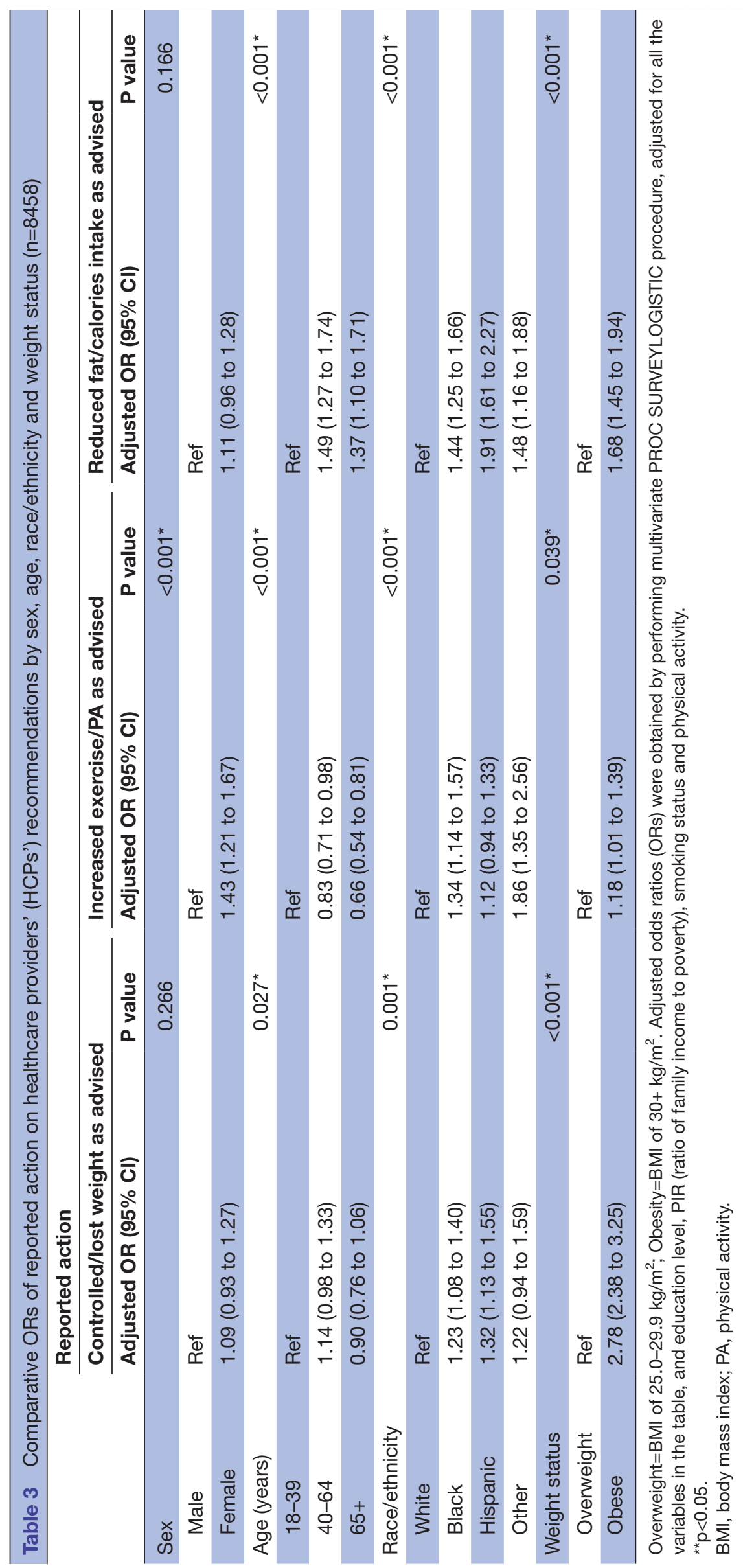


encounter, and providing patients with exercise 'prescriptions' or referrals. ${ }^{52}$ Relatedly, a recent study found that only $10.9 \%$ of adults with overweight/obesity who were advised to lose weight sought help from health professionals. ${ }^{53}$ HCPs also should discuss evidence-based strategies for behaviour change such as self-monitoring, ${ }^{54}$ using SMART (specific, measurable, attainable, relevant and timely) goals, ${ }^{54}$ and seeking social support ${ }^{55}$ for their behaviour change efforts.

Study findings should be considered in light of study limitations, which include the cross-sectional study design and the use of self-reported data assessing receipt of HCP counselling, reported actions on HCPs' recommendations and PA levels. Furthermore, the NHANES data set does not provide details about the scope and frequency of HCP counselling. Future research could use qualitative research or mixed methods to explore both patients' and HCPS' perspectives about HCP counselling and adoption of HCPs' recommendation in depth while longitudinal studies using objective measures could determine if the adoption of HCP recommendations resulted in increased PA, changes in diet and weight loss. Lastly, it is possible that there is response bias for the reported adoption of HCPs' recommendations. Study strengths include examining three different recommendations and adoption of these recommendations, although the recommendation assessing calorie/fat intake did not assess type of fat (saturated, unsaturated). Additional study strengths include a large sample size and use of a national data set that included measured height and weight which was used to determine weight status. Study results are generalisable to non-institutionalised civilian adults aged $18+$ in the US due to the NHANES' sampling frame.

\section{Conclusion}

The results of this analysis highlight several important aspects of recommendations for weight management, PA and diet among adults in the US who are overweight or have obesity. First, there is a need for more patients who are overweight or have obesity to receive HCP counselling related to losing/controlling weight, increasing exercise/ PA and changing caloric and fat intake. Additionally, there is a need for HCPs to provide counselling to individuals who are overweight to potentially decrease the likelihood for transitioning to obesity. HCPs likely need additional training on how best to address behaviour change with patients. As differences in HCP counselling were identified by sex, age and race/ethnicity, there is a critical need to address these differences. Since many patients did not take action on HCPs' recommendations, efforts should be undertaken to increase adoption of offered recommendations. These efforts could include HCPs discussing evidencebased strategies for behaviour change with patients and providing patients with appropriate referrals to promote increased adoption of offered recommendations.

Acknowledgements This work uses data provided by NHANES and would not have been possible without access to this data. We also would like to thank the NHANES respondents for their time and effort
Contributors MG and FX conceptualised the study. MG, FX and SC and defined the analysis. FX performed data analysis with the assistance of SC. MG drafted the manuscript. FX, SC, CRW-R and DR contributed to the drafting and revising of the manuscript. All authors read, commented and approved the final manuscript.

Funding The authors have not declared a specific grant for this research from any funding agency in the public, commercial or not-for-profit sectors.

Competing interests None declared.

Patient consent for publication Not required.

Ethics approval The study was approved by the Institutional Review Board (IRB) at the University of Rhode Island and classified as exempt (IRB\#: 1544383-1).

Provenance and peer review Not commissioned; externally peer reviewed.

Data availability statement Data are available in a public, open access repository. The NHANEs data are available on the NHANES web site. The statistical code and dataset for this paper are available from https://wwwn.cdc.gov/nchs/nhanes/ continuousnhanes/default.aspx.

Supplemental material This content has been supplied by the author(s). It has not been vetted by BMJ Publishing Group Limited (BMJ) and may not have been peer-reviewed. Any opinions or recommendations discussed are solely those of the author(s) and are not endorsed by BMJ. BMJ disclaims all liability and responsibility arising from any reliance placed on the content. Where the content includes any translated material, BMJ does not warrant the accuracy and reliability of the translations (including but not limited to local regulations, clinical guidelines, terminology, drug names and drug dosages), and is not responsible for any error and/or omissions arising from translation and adaptation or otherwise.

Open access This is an open access article distributed in accordance with the Creative Commons Attribution Non Commercial (CC BY-NC 4.0) license, which permits others to distribute, remix, adapt, build upon this work non-commercially, and license their derivative works on different terms, provided the original work is properly cited, appropriate credit is given, any changes made indicated, and the use is non-commercial. See: http://creativecommons.org/licenses/by-nc/4.0/.

ORCID iD

Mary L. Greaney http://orcid.org/0000-0002-5963-920X

\section{REFERENCES}

1 Fryar CD, Carroll MD, Ogden CL. Prevalence of overweight, obesity, and severe obesity among adults aged 20 and over: United States, 1960-1962 through 2015-2016. National Center for Health Statistics Health E-Stats, 2018.

$2 \mathrm{NIH}$. The practical guide. In: Identification, evaluation, and treatment of overweight and obesity in adults. Initiative NOE ed. Washington, DC: National Heart, Lung, and Blood Institute, 2000.

3 Abdelaal M, le Roux CW, Docherty NG. Morbidity and mortality associated with obesity. Ann Transl Med 2017;5:161-73.

4 Pool AC, Kraschnewski JL, Cover LA, et al. The impact of physician weight discussion on weight loss in US adults. Obes Res Clin Pract 2014;8:e131-9.

5 Sciamanna CN, Tate DF, Lang W, et al. Who reports receiving advice to lose weight? results from a multistate survey. Arch Intern Med 2000;160:2334-9.

6 Ko JY, Brown DR, Galuska DA, et al. Weight loss advice U.S. obese adults receive from health care professionals. Prev Med 2008;47:587-92.

7 Bleich SN. Pickett-Blakely, \& Cooper LA. Physician practice patterns of obesity diagnosis and weight-related counseling. Patient Educ Couns 2011;82:123-9.

8 Lorts C, Ohri-Vachaspati P. Disparities in who receives weight-loss advice from a health care provider: does income make a difference? Prev Chronic Dis 2016;13:E142.

9 Jackson JE, Doescher MP, Saver BG, et al. Trends in professional advice to lose weight among obese adults, 1994 to 2000. J Gen Intern Med 2005;20:814-8.

10 Ahmed NU, Delgado M, Saxena A. Trends and disparities in the prevalence of physicians' counseling on diet and nutrition among the U.S. adult population, 2000-2011. Prev Med 2016;89:70-5.

11 Paixão C, Dias CM, Jorge R, et al. Successful weight loss maintenance: a systematic review of weight control registries. Obes Rev 2020;21:e13003.

12 Santos I, Vieira PN, Silva MN, et al. Weight control behaviors of highly successful weight loss maintainers: the Portuguese weight control registry. J Behav Med 2017;40:366-71. 
13 Norris SL, Grothaus LC, Buchner DM, et al. Effectiveness of physician-based assessment and counseling for exercise in a staff model HMO. Prev Med 2000;30:513-23.

14 Calfas KJ, Long BJ, Sallis JF, et al. A controlled trial of physician counseling to promote the adoption of physical activity. Prev Med 1996;25:225-33.

15 Patnode CD, Evans CV, Senger CA, et al. Behavioral counseling to promote a healthful diet and physical activity for cardiovascular disease prevention in adults without known cardiovascular disease risk factors: updated evidence report and systematic review for the US preventive services Task force. JAMA 2017;318:175-193.

16 Ahmed NU, Delgado M, Saxena A. Trends and disparities in the prevalence of physicians' counseling on exercise among the U.S. adult population, 2000-2010. Prev Med 2017;99:1-6.

17 Barnes PM, Schoenborn CA. Trends in adults receiving a recommendation for exercise or other physical activity from a physician or other health professional. . NCHS Data Brief, 2012: 86. 1-8.

18 Koopmans GT, Lamers LM. Gender and health care utilization: the role of mental distress and help-seeking propensity. Soc Sci Med 2007;64:1216-30.

19 Loureiro ML, Nayga RM. Obesity, weight loss, and physician's advice. Soc Sci Med 2006:62:2458-68.

20 Bish CL, Blanck HM, Serdula MK, et al. Diet and physical activity behaviors among Americans trying to lose weight: 2000 behavioral risk factor surveillance system. Obes Res 2005;13:596-607.

21 Mogre V, Wanaba P, Apala P, et al. Self-Reported receipt of healthcare professional's weight management counselling is associated with self-reported weight management behaviours of type 2 diabetes mellitus patients. Springerplus 2016;5:379.

22 Centers for Disease Control and Prevention. National health and nutrition examination survey. Available: https://wwwn.cdc.gov/nchs/ nhanes [Accessed 21 Dec 2019].

23 Centers for Disease Control and Prevention. How to measure and interpret weight status. Available: https://www.cdc.gov/ healthyweight/assessing/index.html [Accessed 11 Sep 2020].

24 Armstrong T, Bull F. Development of the world Health organization global physical activity questionnaire (GPAQ). J Public Health 2006;14:66-70.

25 US Department of Health and Human Services. Physical activity guidelines for Americans. 2nd ed. Washington, DC: US Department of Health and Human Services, 2018.

26 CDC National Center for Health Statistics. Specifying weighting parameters, 2013. Available: https://wwwn.cdc.gov/nchs/nhanes/ tutorials/Module3.aspx [Accessed 20 Aug 2020].

27 Miles A, Simon A, Wardle J. Answering patient questions about the role lifestyle factors play in cancer onset and recurrence: what do health care professionals say? J Health Psychol 2010;15:291-8.

28 Alexander SC, Cox ME, Yancy WS, et al. Weight-loss talks: what works (and what doesn't). J Fam Pract 2011;60:213-9.

29 Guglielmo D, Hootman JM, Murphy LB, et al. Health Care Provider Counseling for Weight Loss Among Adults with Arthritis and Overweight or Obesity - United States, 2002-2014. MMWR Morb Mortal Wkly Rep 2018;67:485-90.

30 Vetter ML, Herring SJ, Sood M, et al. What do resident physicians know about nutrition? an evaluation of attitudes, self-perceived proficiency and knowledge. J Am Coll Nutr 2008;27:287-98.

31 Petrin C, Kahan S, Turner M, et al. Current attitudes and practices of obesity counselling by health care providers. Obes Res Clin Pract 2017;11:352-9.

32 Manuel Jl. Racial/Ethnic and gender disparities in health care use and access. Health Serv Res 2018;53:1407-29.

33 Honda K. Factors underlying variation in receipt of physician advice on diet and exercise: applications of the behavioral model of health care utilization. Am J Health Promot 2004;18:370-7.

34 Eaton CB, Goodwin MA, Stange KC. Direct observation of nutrition counseling in community family practice. Am J Prev Med 2002;23:174-9.
35 Lorts C, Adams MA, Tasevska N, et al. Community food environment moderates association between health care provider advice to lose weight and eating behaviors. Prev Med Rep 2019;15:100926.

36 Artaud F, Sabia S, Dugravot A, et al. Trajectories of unhealthy behaviors in midlife and risk of disability at older ages in the Whitehall II cohort study. J Gerontol A Biol Sci Med Sci 2016;71:1500-6.

37 Dorner TE, Rieder A. Obesity paradox in elderly patients with cardiovascular diseases. Int J Cardiol 2012;155:56-65.

38 Oreopoulos A, Kalantar-Zadeh K, Sharma AM, et al. The obesity paradox in the elderly: potential mechanisms and clinical implications. Clin Geriatr Med 2009;25:643-59.

39 Hales CM, Carroll MD, Fryar CD, et al. Prevalence of obesity among adults and youth: United States, 2015-2016. NCHS data brief, no 288. Hyattsville, MD: National Center for Health Statistics, 2017.

40 Sallis R, Franklin B, Joy L, et al. Strategies for promoting physical activity in clinical practice. Prog Cardiovasc Dis 2015;57:375-86.

41 Block JP, DeSalvo KB, Fisher WP. Are physicians equipped to address the obesity epidemic? knowledge and attitudes of internal medicine residents. Prev Med 2003;36:669-75.

42 Kolasa KM, Rickett K. Barriers to providing nutrition counseling cited by physicians. Nutr Clin Pract 2010;25:502-9.

43 Forman-Hoffman V, Little A, Wahls T. Barriers to obesity management: a pilot study of primary care clinicians. BMC Fam Pract 2006;7:35.

44 Huang J, Yu H, Marin E, et al. Physicians' weight loss counseling in two public hospital primary care clinics. Acad Med 2004;79:156-61.

45 Kushner RF. Barriers to providing nutrition counseling by physicians: a survey of primary care practitioners. Prev Med 1995;24:546-52.

46 Butsch WS, Kushner RF, Alford S, et al. Low priority of obesity education leads to lack of medical students' preparedness to effectively treat patients with obesity: results from the U.S. medical school obesity education curriculum benchmark study. BMC Med Educ 2020;20:23.

47 Bleich SN, Bandara S, Bennett WL, et al. U.S. health professionals views on obesity care, training, and self-efficacy. Am J Prev Med 2015;48:411-8.

48 Royal College of Physicians. The training of health professionals for the prevention and treatment of overweight and obesity 2010 report prepared for Foresight by the Royal College of clinicians. Available: www.rcplondon.ac.uk/file/268 [Accessed 25 May 2020].

49 Foster GD, Wadden TA, Makris AP, et al. Primary care physicians' attitudes about obesity and its treatment. Obes Res 2003;11:1168-77.

50 Talking with Patients about Weight Loss. Tips for Primary Care Providers National Institute of Diabetes and Digestive and kidney Diseases, 2017. Available: https://www.niddk.nih.gov/healthinformation/weight-management/talking-adult-patients-tips-primarycare-clinicians [Accessed 13 Sep 2020].

51 Aveyard P, Lewis A, Tearne S, et al. Screening and brief intervention for obesity in primary care: a parallel, two-arm, randomised trial. Lancet 2016;388:2492-500.

52 Exercise is Medicine Framework. Health care providers. Available: https://www.exerciseismedicine.org/support_page.php/health-careproviders/ [Accessed 13 Sep 2020].

53 de Heer Hendrik 'Dirk', Kinslow B, Lane T, et al. Only 1 in 10 patients told to lose weight seek help from a health professional: a nationally representative sample. Am J Health Promot 2019;33:1049-52.

54 Hooker S, Punjabi A, Justesen K, Sherman MD, et al. Encouraging health behavior change: eight evidence-based strategies. Fam Pract Manag 2018;25:31-6.

55 Greaney ML, Puleo E, Sprunck-Harrild K, Houghton S, et al. Social support for changing multiple behaviors: factors associated with seeking support and the impact of offered support. Health Educ Behav 2018;45:198-206. 